\title{
BMJ Open Telehealth voice assessment by speech language pathologists during a global pandemic using principles of a primary contact model: an observational cohort study protocol
}

\author{
Christopher L Payten (D) , , Duy Duong Nguyen (D) , ${ }^{2}$ Daniel Novakovic (1) , ${ }^{2}$ \\ John O'Neill, ${ }^{3}$ Antonia M Chacon (I) , ${ }^{2}$ Kelly A Weir (1) , ${ }^{4,5}$ Catherine J Madill (1) ${ }^{2}$
}

To cite: Payten CL, Nguyen DD, Novakovic D, et al. Telehealth voice assessment by speech language pathologists during a global pandemic using principles of a primary contact model: an observational cohort study protocol. BMJ Open 2022;12:e052518. doi:10.1136/ bmjopen-2021-052518

- Prepublication history and additional supplemental material for this paper are available online. To view these files, please visit the journal online (http://dx.doi.org/10.1136/ bmjopen-2021-052518)

Received 18 April 2021 Accepted 16 December 2021

Deck for updates

(c) Author(s) (or their employer(s)) 2022. Re-use permitted under CC BY-NC. No commercial re-use. See rights and permissions. Published by BMJ.

For numbered affiliations see end of article.

\section{Correspondence to}

Christopher L Payten; christopher.payten@health.qld. gov.au

\section{ABSTRACT}

Introduction SARS-CoV-2, a highly contagious severe acute respiratory syndrome, has spread to most countries in the world and resulted in a change to practice patterns for the assessment and diagnosis of people with voice disorders. Many services are transitioning to telehealth models to maintain physical distancing measures and conserve personal protective equipment used by healthcare workers during laryngoscopy examinations. The speech-language pathology primary contact (SLPPC) assessment for patients referred to ear, nose and throat (ENT) services in Australia has been shown to reduce waiting times for assessment while streamlining access to ENT assessment and allied health practitioner treatment pathways.

Methods and analysis A prospective observational cohort study will see patients in a newly developed telehealth model which uses the principles from a usual care SLPPC assessment protocol. Participants will be offered an initial telehealth assessment (speech-language pathology primary contact telehealth (SLPPC-T)) prior to being prioritised for a face-to-face laryngoscopy assessment to complete the diagnostic process. The telehealth assessment will collect sociodemographic information, personal and family medical history, key symptoms, onset and variability of symptoms, redflag signs or symptoms for laryngeal malignancy, and clinical voice assessment data for auditory-perceptual and acoustic analysis. The study outcomes include (1) association of signs, symptoms and specific voice measures collected during SLPPC-T with voice disorder classification provided after laryngoscopy; (2) degree of concordance between voice disorder classification after SLPPC-T and after laryngoscopy; (3) health service and patient-related costs and health outcomes of the SLPPC-T; (4) patient and stakeholder views and beliefs about the SLPPC-T process.

Ethics and dissemination Ethical approval has been granted prior to commencement of the study enrolment by the Gold Coast Hospital and Health Service Human Research Ethics Committee (reference number HREC/2020/QGC/62832). Results will be shared through the publication of articles in peer-reviewed medical

\section{Strengths and limitations of this study}

- This study has been developed in response to the current SARS-CoV-2 global pandemic where access to face-to-face instrumental assessments of vocal symptoms is being prioritised and deferred.

- This is the first study to examine the impact of a speech-language pathology primary contact (SLPPC) telehealth voice assessment as a way of triaging the priority for ear, nose and throat (ENT) assessment and intervention.

- The telehealth model is based on principles of a face-to-face speech-language pathology primary contact assessment model which has been shown to improve waiting times and access to diagnostic assessments with both ENT surgeons and speech language pathologists (SLP).

- Although the study relies on a telehealth assessment model which may not be accessible for all patients, we have designed the protocol so that data can be collected using a hybrid assessment approach including a mix of telehealth, written communication and face-to-face consultations.

- The SLPPC assessment pathway is not readily available in Australia and may not be a viable model for all health services. However, the protocol is designed so that both SLPs and ENTs, or a combination, can collect assessment data in the same way to facilitate a triage process.

journals and presentation at national and international scientific meetings.

Trial registration number ACTRN12621000427875.

\section{INTRODUCTION}

COVID-19 (WHO's terminology for 'coronavirus disease 2019') refers to a severe acute respiratory syndrome caused by a newly identified coronavirus strain (SARS-CoV-2). COVID-19 has spread rapidly to most countries and territories across the world since its 
first outbreak in Wuhan City (Hubei Province, China). ${ }^{1}$ As of 18 April 2021, over 130 million people have contracted the virus resulting in almost three million deaths. ${ }^{2}$ In an attempt to control the spread of the virus, governments have enforced strict social distancing measures and travel restrictions. ${ }^{3}$ There is a reported increase in psychological distress worldwide. ${ }^{4}$ The financial impact of the pandemic is also extensive. ${ }^{5}$

Although the incidence of the disease varies across different countries and regions, ${ }^{6}$ and its mortality rate is generally lower than that of previous SARS-CoV-2 outbreaks, ${ }^{7}$ the impact of the COVID-19 pandemic on the world's health system is hugely significant. ${ }^{8-10}$ The situation is rapidly changing with reports of new variants of SARS-CoV-2 with reported increased rates of transmission by up to $70 \% .{ }^{11}$ Although a number of treatment methods have been suggested, there has been a lack of scientific evidence confirming the effectiveness of those methods. ${ }^{12}$ As a result, strategies have been planned to mitigate the spread and impact of the pandemic ${ }^{13}$ and to cope with consequences of the disease ${ }^{14}$ in view that the virus will persist until a global safe and effective strategy for vaccination is implemented successfully. ${ }^{1516}$

As the mode of transmission of COVID-19 is via droplets and airborne aerosols, the risk of a healthcare worker contracting the virus is high. There is preliminary evidence that high viral load occurs in sputum samples followed by nasal and throat samples. ${ }^{1718}$ The clinical practice of ear, nose and throat (ENT) surgeons and speech language pathologists (SLPs) entails a range of tasks and procedures that are categorised as aerosol-generating procedures, including endoscopy for voice and swallowing assessments. ${ }^{19}$ These are believed to involve a high risk of transmission of respiratory infection. ${ }^{20}$ Consequently, advice from multiple ENT and SLP associations during the pandemic, in areas where community transmission is high, is to minimise face-to-face consultations and prioritise endoscopic examinations to urgent cases while employing strict conditions to minimise transmission risk. $^{21-23}$

The prevalence of voice disorders in the Australian general population is estimated to be at least $6.8 \% .^{24}$ Typically, patients with a voice disorder may experience symptoms of altered vocal quality, pitch, loudness, flexibility or effort when compared with someone of the same gender, age or cultural background. ${ }^{25}$ Living with a voice disorder has been shown to impact an individual's physical, emotional and employment well-being and incur major costs to healthcare and society. ${ }^{26-28}$ Early assessment and intervention are therefore important to prevent vocal decline and reduce healthcare costs. ${ }^{29}$ Voice disorders encompass a wide variety of different conditions with signs and symptoms that can present in isolation or combination with each other. Hoarseness may be a symptom of an underlying acute or progressive medical condition. ${ }^{30}$ However, around $40 \%$ of patients are diagnosed with a voice disorder where there is no organic pathology nor neuromuscular pathophysiology. ${ }^{31}$
In comparison, the incidence of unilateral vocal fold palsy or paresis is $1.2 \%,{ }^{32}$ and a reported $2.2 \%$ of people seeking treatment for vocal symptoms are diagnosed with laryngeal cancer. ${ }^{33}$ Visualisation of the larynx using laryngoscopy is the recommended assessment to identify organic pathology. ${ }^{30}$ However, classification and diagnosis beyond that of organic laryngeal pathology require a multidisciplinary and multidimensional assessment including a range of clinical voice measures to encapsulate the complexity of the vocal mechanism..$^{30} 34$ In the assessment pathway for voice-disordered patients, SLP assessment includes perceptual and instrumental voice evaluation, which is considered as having a lower risk of virus exposure than ENT evaluation but provides important information for diagnosis and successful treatment of a voice disorder. ${ }^{34} 35$

The current pandemic has highlighted several threats to ENT and SLP services for patients with vocal symptoms when access to laryngoscopy is deferred. ${ }^{36}$ This includes delayed diagnosis of head and neck cancers, delayed or reduced access to instrumental procedures, and inequalities in the provision of care. ${ }^{36}$ In response to these threats, opportunities for new models of care are being explored. ${ }^{36}$ For example, an evidenced-based telephone triage system in the UK has been used to effectively identify patients with probable cancer according to their risk factors identified through case history. ${ }^{37}$

In usual circumstances, the 'gold standard' first contact assessment of vocal symptoms is laryngoscopy with ENT, preferably in an interdisciplinary clinical model with both ENT surgeon and SLP present. ${ }^{29}$ Allied health practitioner (AHP) expanded scope primary contact pathways are an emerging alternative to the traditional medical-led first contact pathways, providing high value and lower-cost care in areas where AHP treatment pathways exist. ${ }^{38-41}$ One example is the speech-language pathology primary contact (SLPPC) model of voice assessment where patients are seen in a parallel clinic to ENT for face-to-face assessment by SLPs including case history, clinical voice assessment and direct laryngoscopy with stroboscopy, prior to case discussion with the ENT for diagnosis and treatment planning ${ }^{42-44}$ (figure 1). These models provide a more efficient pathway for patients by reducing wait times for assessment and providing faster access to both ENT and voice treatment services, when compared with the ENTled models. ${ }^{42-44}$ A recently published retrospective 5-year audit of a SLP led first contact clinic has confirmed the safety and service benefits of this model, including only a $2 \%$ re-referral rate over 5 years. ${ }^{45}$ Whereas up to $80 \%$ of patients seen in these pathways can be managed by SLPs after diagnosis, around $7 \%$ are identified for more urgent ENT assessment, which may include further diagnostic tests (eg, radiology). ${ }^{44}$ The face-to-face SLP-led model relies on concurrent timetabling of SLP and ENT clinics, and a potential overuse of the ENT surgeons' time and resources for $80 \%$ of patients. A remote SLPPC assessment may be considered a valuable step in the triageassessment sequence for diagnosis of a voice disorder. 


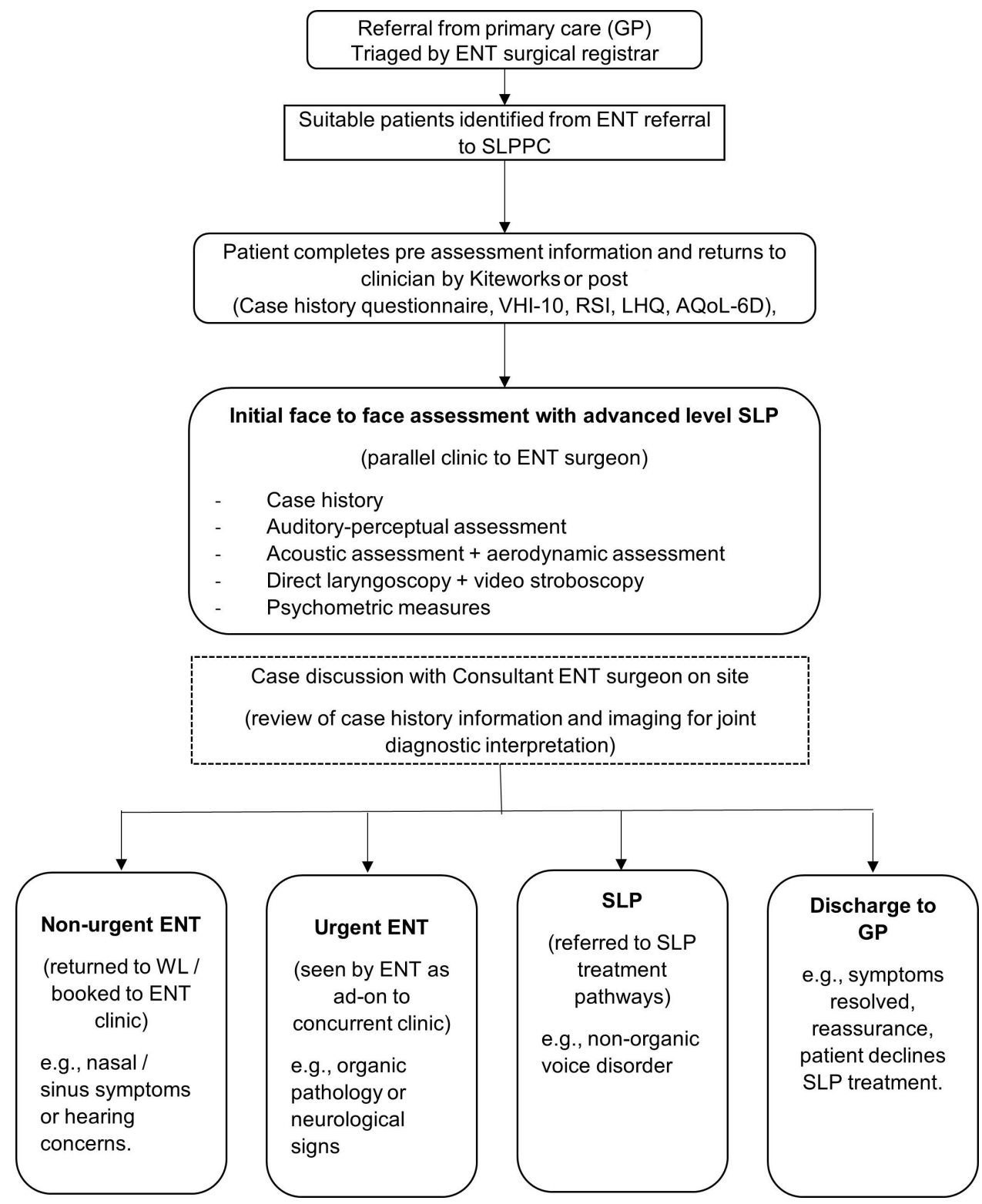

Figure 1 Care pathway for the 'usual care' SLPPC at Gold Coast Hospital and Health Service (GCHHS). AQoL-6D, Assessment of Quality of Life-6D; ENT, ear, nose and throat; GP, general practitioner; LHQ, Laryngeal Hypersensitivity Questionnaire; RSI, Reflux Symptom Index; SLP, speech-language pathologist; SLPPC, speech-language pathology primary contact; VHI-10, Voice Handicap Index-10; WL, ENT waitlist.

SLPs can use their expertise and skills to document signs and symptoms through detailed case histories and auditory-perceptual and acoustic voice evaluation. Skilled and high-quality data collection may help facilitate early identification of patients who need escalation to an ENT before they attend the hospital for laryngoscopy, enabling more targeted appointments in the right clinic with the right professional. Less critical patients can then be seen for routine assessment by the SLP, with a brief ENT case review to confirm the laryngeal diagnosis prior to commencement of SLP voice treatment.

Telehealth is an effective model used by SLPs and ENTs as an alternative to face-to-face consults to diagnose and treat voice disorders in remote settings. ${ }^{46}$ In the Australian context, validity and reliability of the telehealth assessment model in the Parkinson's disease population have comparable results to in-person consults for perceptual and acoustic measures of voice and speech. ${ }^{47}$ In the USA, the use of telehealth consultations by SLPs has been shown to contribute to medical diagnosis for a range of voice disorders with equal diagnostic decision outcomes to in-person consultations, including recommendations for specialist ENT intervention. ${ }^{48}$ Furthermore, significantly comparable outcomes have been demonstrated in auditory-perceptual evaluations, acoustic measures, patient satisfaction and laryngoscopy ratings between a telehealth delivery model compared with a face-to-face pathway. ${ }^{49}$ This rapidly developing area of practice which improves access to specialist healthcare for people in remote areas and better collaboration between specialists 
is of relevance to the SLPPC model, particularly in the wake of the COVID-19 pandemic, where access to diagnostic laryngoscopy may be limited or delayed.

\section{Aims}

The aim of this study is to examine the speech-language pathology primary contact telehealth (SLPPC-T) assessment model as a remote triage assessment to identify a core set of clinical measures which can identify participants suitable for an SLP-led treatment model and those who need an ENT or interdisciplinary assessment model. A range of measures obtained through a virtual assessment protocol will be examined against the voice disorder classification provided after laryngoscopy to determine the influence of these measures on priority for ENT examination.

\section{METHODS AND ANALYSIS Objectives}

The primary objective is to examine the predictive value of signs, symptoms and voice measures collected during the SLPPC-T assessment on the voice disorder classification provided by ENT after laryngoscopy using a linear regression model.

The secondary objectives are to

1. Examine the agreement in voice disorder classification provided by SLPs after remote triage assessment and voice disorder classification provided by ENT after laryngoscopy, using statistical measures of concordance.

2. Examine the agreement in voice disorder classification provided by SLPs after laryngoscopy and voice disorder classification provided by ENT after laryngoscopy, using statistical measures of concordance.

3. Examine patient views on the SLPPC-T model using quantitative and qualitative analysis of survey data.

4. Examine patient and health service attributed costs associated with the SLPPC-T model.

\section{Study design}

This is a prospective observational cohort study using principles from the SLPPC assessment protocol at Gold Coast University Hospital (GCUH), as previously described. ${ }^{44}$ All participants triaged to the SLPPC clinic will be offered an initial telehealth assessment prior to being prioritised for a face-to-face laryngoscopy and ENT diagnosis to complete the diagnostic process.

\section{Participants}

New patients referred for initial ENT evaluation who are triaged by the ENT surgical team as category 2 (complex care, assessment for $<90$ days) or category 3 (non-urgent, delayed assessment unlikely to lead to deterioration or a need for more complex care, assessment for $<365$ days), in accordance with the Queensland Health Clinical Prioritisation Criteria (CPC) ${ }^{50}$ The SLPPC assessment clinic receives approximately five referrals per week, enrolment will continue over a period of 12-18 months to facilitate recruitment of enough participants for this study.

Inclusion criteria

- Adults aged over 18.

- Episodes of hoarseness or altered voice in patients with no risk factors for malignancy identified on written referral.

- Patients who can provide informed consent and complete a telehealth case history and assessment in English.

\section{Exclusion criteria}

- Patients whose referrals are triaged by ENT as category 1 based on written referral information, as defined by the Queensland Health CPC. ${ }^{50}$

- Any significant medical comorbidities identified on written referral (ie, previous head and neck cancer, previous laryngeal surgery and neuromuscular disease affecting the larynx).

\section{Sample size}

A power calculation was completed to consider a model examining the relationship between signs, symptoms and voice measures and diagnostic group membership, with reference to studies in the literature that have examined characteristics of a general voice-disordered population. ${ }^{51}{ }^{52}$ Based on these data, with $\alpha=0.05$ and power of $90 \%, 150$ participants were identified as the minimum appropriate sample size to complete the logistic regression analysis.

For the concordance analysis, sample size was calculated using guidelines in the literature on a calculation of minimum sample size for Cohen's kappa ${ }^{53}$ Calculations were made using a $5 \times 5$ category table where each category (voice disorder diagnostic group) is assumed proportionate and based on power at $90 \%$ and $\alpha=0.05$. The minimum sample size for this analysis has been calculated at 55 individual participants.

Given the power calculations for the regression analysis outcome measure is greatest, the minimum sample size for this study is at 150 .

\section{Setting}

The study is sponsored by the Gold Coast Hospital and Health Service (GCHHS). The sponsor had no role in the design of this study and will not have any role during the study execution, analyses, data interpretation or a decision to submit results. Potential participants will be recruited from GCUH. Data will be analysed by the principal investigator and coinvestigators at GCHHS, The University of Sydney and Griffith University.

\section{Interventions}

The telehealth assessment protocol consists of multiple parts designed to collect information relevant to the patients' presenting vocal symptoms including sociodemographic information, personal and family medical history, key symptoms, onset and variability of symptoms, 


\begin{tabular}{|c|c|}
\hline \multicolumn{2}{|r|}{ Indications for priority laryngoscopy after SLPPC telehealth assessment } \\
\hline Level & Criteria \\
\hline $\begin{array}{c}\text { Emergent } \\
\text { laryngoscopy }\end{array}$ & $\begin{array}{l}\text { Hoarseness in combination with other significant red flag symptoms } \\
\text { including dysphagia, odynophagia, dyspnoea, }>10 \% \text { weight loss. } \\
\text { - Where airway patency is a concern. } \\
\text { - Existing or likely vocal fold palsy where aspiration may be a concern. }\end{array}$ \\
\hline $\begin{array}{l}\text { High priority } \\
\text { laryngoscopy }\end{array}$ & $\begin{array}{l}\text { - Aphonia / severely dysphonic patients where previous laryngoscopy has not } \\
\text { been completed. } \\
\text { - Clinical symptoms and signs where organic laryngeal pathology is } \\
\text { suspected (i.e., fluctuating vocal quality of roughness and breathiness + } \\
\text { limited response to a trial of voice therapy, symptoms of laryngeal soreness, } \\
\text { sustained lowered habitual pitch). } \\
\text { - Clinical symptoms and signs where neuromuscular aetiology is suspected } \\
\text { (i.e., sustained breathy or asthenic vocal quality with limited response to a } \\
\text { trial of voice therapy, symptoms of laryngeal +/- other neurological signs or } \\
\text { symptoms). } \\
\text { - Dysphonia with high level of patient concern. } \\
\text { - Deterioration in vocal symptoms or poor response to a trial of voice therapy. }\end{array}$ \\
\hline $\begin{array}{c}\text { Routine } \\
\text { laryngoscopy }\end{array}$ & $\begin{array}{l}\text { Dysphonia with low level patient concern where previous laryngoscopy has } \\
\text { not been completed. } \\
\text { Clinical symptoms and signs where no organic laryngeal pathology is } \\
\text { suspected (i.e., less fluctuating vocal quality of roughness and breathiness, } \\
\text { perceptual judgements of strained vocal quality + good / excellent response } \\
\text { to a trial of voice therapy, symptoms of laryngeal soreness, sustained } \\
\text { lowered habitual pitch). } \\
\text { - Improved vocal symptoms with a trial of voice therapy. }\end{array}$ \\
\hline
\end{tabular}

Figure 2 Priority matrix for how patients will be triaged for urgency of laryngoscopy after telehealth triage assessment. ENT, ear, nose and throat; SLP, speech-language pathologist; SLPPC, speech-language pathology primary contact.

red-flag signs or symptoms for laryngeal malignancy, and clinical voice assessment data for auditory-perceptual and acoustic analysis. These assessments make up the voice assessment protocol in a face-to-face SLPPC service at GCUH. The only assessment not able to be provided by telehealth is laryngoscopy, which will be provided for all participants at a face-to-face appointment in the SLPPC clinic or interdisciplinary voice clinic once priority for laryngoscopy is determined.

To obtain remote voice samples for the auditoryperceptual and acoustic analysis, participants will be given written and verbal guidance by the clinician at the end of the telehealth assessment. Voice samples will be collected from the subject's home computer or laptop using the free to download voice recording software (Audacity) or a mobile phone application (AVR-X for iPhone or Smart Voice recorder for Samsung Galaxy). These apps are free to download, allow sharing of recorded .wav files and offer a sampling rate $44100 \mathrm{~Hz}$ for recording. Participants will be instructed to use a headset mic (where possible) and to set the mic to mouth distance at $5 \mathrm{~cm}$ for the recording. To account for the effect of the home recording environment, participants will be asked to record a $5 \mathrm{~s}$ period of silence and to measure the level of background noise (SPL, $\mathrm{dB}$ ) using a free to download smartphone app. Where a home recording cannot be provided by the participant, a voice sample will be collected at the face-to-face assessment using a headmounted condenser microphone with flat frequency response (Shure Beta53) at a mouth-mic distance of $5 \mathrm{~cm}$ recorded into Audacity.

Acoustic analysis will be completed on the voice samples using Praat free software. ${ }^{54}$ The measures of interest will include time-based measures of fundamental frequency and amplitude from a sustained vowel, and smoothed cepstral peak prominence measures of connected speech. Assessment of acoustic signal typing will be completed on the sustained vowel samples to ensure the signal is suitable for acoustic analysis of harmonics to noise ratio. ${ }^{55}$

After SLPPC-T assessment, participants will be prioritised for laryngoscopy and completion diagnosis by an ENT, as follows:

1. Emergency time-sensitive laryngoscopy by ENT, completed within 5 days.

2. Urgent high-priority laryngoscopy with ENT present during the laryngoscopy or at an interdisciplinary clinic, within 2 weeks.

3. Routine laryngoscopy in the SLPPC clinic parallel to ENT within 2-4 weeks. Case discussion and a joint review of the assessment data, including laryngeal imaging, will be completed on the same day at the end of the clinic.

Figure 2 shows the priority matrix used to guide how participants will be triaged for urgency of laryngoscopy. 


\section{Primary outcome measure}

The primary outcome measure is the association of signs, symptoms and specific voice measures collected during the SLPPC-T assessment with voice disorder classification category provided after laryngoscopy.

The primary outcome measure will be analysed by multivariate logistic regression analysis.

Variables will include

1. Case history information as reported through a preassessment written case history questionnaire and during the telehealth assessment (SLPPC-T):

- Presenting vocal/laryngeal symptoms.

- Onset, variability and progression of vocal/laryngeal symptoms.

- Medical, surgical, family and medication history.

- Presence of coexisting symptoms (airway, cough, swallowing, globus, odynophagia, unexplained weight loss, unilateral otalgia, unilateral neck lump, unexplained night sweats or fevers and haemoptysis).

- Presence of contributing or aggravating symptoms (reflux, neck/shoulder tension, stress, degree of voice use and effect of alcohol).

- Voice use history.

- A subset of questions from the Head and Neck Cancer Risk Calculator V.2 published by the Royal College of Surgeons of England as part of a 2-week wait telephone triage system. ${ }^{56}$

2. Voice aerodynamic measures of maximum phonation time in seconds and $\mathrm{S} / \mathrm{Z}$ ratio (time difference between a participant's maximum sustained 's' and ' $z$ ') collected during the SLPPC-T.

3. Perceptual voice quality measures using the Consensus Auditory-Perceptual Evaluation of Voice (CAPE-V) ${ }^{57}$ (using a visual analogue scale from 0 (normal) to 100 (severe)) to evaluate grade, overall perceived voice quality severity; rough, degree of roughness or harshness; breathy, degree of breathiness; and strain, degree of strain. Voice recordings will be obtained at baseline during the SLPPC-T assessment using the participants' home device or when they attend the clinic for laryngoscopy assessment.

4. Acoustic voice quality measures captured using the participants home recording or the in-clinic recording and analysed using Praat. $^{54}$ The noiseto-harmonic ratio, mean fundamental frequency, pitch range, dynamic range, smoothed cepstral peak prominence, pitch range and dynamic range data will be collected at baseline from the voice recordings obtained.

5. Task stimulability of voice therapy techniques measured as excellent, good or poor.

6. Voice-related quality of life measured using the validated Voice Handicap Index-10 (VHI-10). ${ }^{58}$

7. Self-reported symptoms of laryngopharyngeal reflux measured using the validated Reflux Symptom Index (RSI). ${ }^{59}$
8. Self-reported symptoms of laryngeal hypersensitivity measured using the validated Newcastle Laryngeal Hypersensitivity Questionnaire (LHQ) ${ }^{60}$

9. Health-related quality of life scores measured using the validated Assessment of Quality of Life-6D (AQoL-6D), a health-related quality of life instrument designed for use with cost-utility analysis. ${ }^{61}$

10. Diagnostic classification impression after SLPPC-T and laryngoscopy, as described by Baker and colleagues: $^{62}$

- Functional neurological (psychogenic) voice disorder.

- Muscle tension voice disorder without suspected/ visible laryngeal pathology.

- Muscle tension voice disorder with suspected/visible laryngeal pathology.

- Organic voice disorder.

- Laryngeal malignancy/airway disorder.

\section{Secondary outcome measures}

The secondary outcome measures include the

1. Degree of concordance between voice disorder classification after SLPPC-T and voice disorder classification after laryngoscopy, measured by statistical measures of agreement (weighted kappa).

2. Degree of concordance between voice disorder classification by SLP after laryngoscopy and voice disorder classification by ENT after laryngoscopy measured by statistical measures of agreement (weighted kappa).

3. Patient views on the SLPPC-T measured using a trialspecific patient experience questionnaire after completion of the in-person visit for laryngoscopy, analysed through quantitative and qualitative survey data analysis.

4. Direct health service costs and direct and indirect patient attributed costs to contribute to a cost-utility analysis of the SLPPC-T (staff costs, equipment costs, AQoL-6D and patient experience surveys).

\section{Participant flow}

The study commenced on 22 May 2021, during the recruitment period of 12-18 months, potential participants will be contacted by the study site administration officer (AO) by telephone and offered a telehealth video assessment in place of a usual care face-to-face assessment. Initial AO telephone consult will include set-up arrangements for video conferencing in accordance with the local study site governance arrangements. Participants will be informed of the study by the AO at this point. The primary contact SLP, Principal Investigator (PI) will screen all referrals to confirm eligibility for the study.

The AO will send information about the telehealth appointment and blank copies of (1) prescreening case history questionnaire; (2) VHI-10, RSI, LHQ and AQoL-6D prior to the consultation. Instructions will be provided for the participant to complete these forms electronically and save them for upload via Kiteworks, a secure file sharing platform approved for clinical use by 


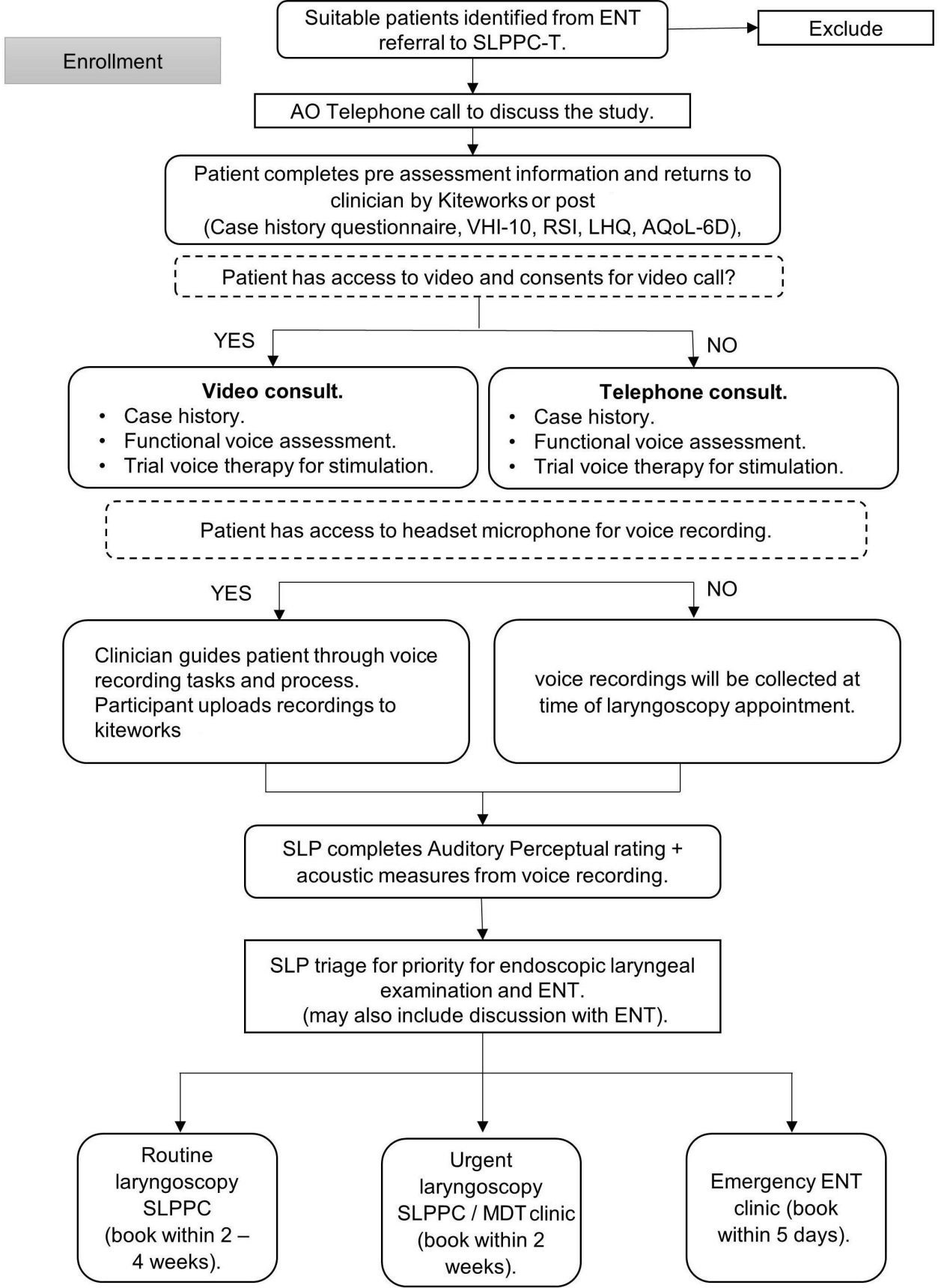

SLP and ENT case discussion and review to confirm diagnosis and treatment recommendations (same day)

Data analysis

Figure 3 The SLPPC-T assessment pathway. AO, administration officer; AQoL-6D, Assessment of Quality of Life-6D; ENT, ear, nose and throat; LHQ, Laryngeal Hypersensitivity Questionnaire; MDT, multidisciplinary team clinic; RSI, Reflux Symptom Index; SLP, speech-language pathologist; SLPPC-T, speech-language pathology primary contact telehealth; VHI-10, Voice Handicap Index-10.

Queensland Health. The AO will contact the participant by telephone to guide them through this process.

The participant information and consent form will be sent to the participant by email or post (if requested) and the PI will be available to answer any questions about the study at the participants request prior to the telehealth assessment. The participant will be asked to complete the consent form and send back to the research team either by Kiteworks or postal return.

During the SLPPC-T triage assessment, the participant will be guided by the clinician to record a standardised voice sample onto their home computer using a free to 
Table 1 Speech-language pathology primary contact telehealth voice assessment protocol

- Preassessment case history questionnaire emailed to the participants for written completion prior to initial telehealth assessment.

- Patient self-report scales

- Voice Handicap Index-10.58

- Reflux Symptom Index. ${ }^{59}$

- Newcastle Laryngeal Hypersensitivity Questionnaire. ${ }^{60}$

- Telehealth case history interview.

- Recording of voice assessment tasks for auditory perceptual and acoustic evaluation. Participants will be guided in how to conduct the recording. Minimum samples will include

- Brief 20-30 s conversation sample.

- Standard reading passage (Rainbow passage). ${ }^{64}$

- Consensus Auditory-Perceptual Evaluation of Voice phrases. ${ }^{57}$

- Prolonged /a:/ vowel at habitual pitch to measure maximum phonation time $(\times 3)$.

- Sustained /s/ and /z/ to measure s:z ratio.

- Loudness (dynamic) range: softest and loudest phonation on prolonged /a:/.

- Pitch range: glide from lowest to highest pitch on /a:/ vowel.

- Counting 1-10 with the patient palpating the larynx.

- Counting eighty to ninety (for voice onset acoustic measures).

- Singing 'Happy Birthday'.

Speech Language Pathology auditory perceptual ratings using the Consensus Auditory-Perceptual Evaluation of Voice rating scale ${ }^{57}$ to include the following measures: nominal score out of 100, categorical scores (mild, moderate, severe), categorical scores (constant or intermittent) to include

1. Overall grade of severity.

$\mathrm{n} / 100$

Mild/moderate/severe

Intermittent/constant

2. Laryngeal roughness.

3. Laryngeal breathiness.

4. Laryngeal strain.

5. Pitch.

6. Loudness.

Task stimulability of voice therapy techniques recorded as excellent, good or poor.

Assessment of Quality of Life-6D. ${ }^{6}$

Locally developed patient-reported experience survey.

Instrumental voice assessment protocol (using the voice recording provided by patient).

Where a home recording has not been provided, measures will be taken from the clinic voice recording at face to face assessment.

Tasks Acoustic measures

Sustained /a:/ vowel for duration of 3-5s Spectographic signal typing: types 1, 2 and 3 (x3) Harmonics to noise ratio (HNR) Vocal pitch SD: $\mathrm{F}_{0}(\mathrm{~Hz})$

Standard reading passage (Rainbow passage) ${ }^{64}$

Mean vocal pitch: average fundamental frequency $\left(\mathrm{F}_{0} \mathrm{~Hz}\right)$

Mean amplitude (dB)

Smoothed cepstral peak prominence (CPPS)

Loudness range: sustained /a:/ vowel at loudest level for duration of $3-5 s$ and at softest level for duration of $3-5 s(x 3)$
Pitch range: sustained /a:/ vowel at lowest pitch for duration of $3-5 s$ and at

highest pitch for duration of $3-5 s(x 3)$

Maximum vocal SPL $(\mathrm{dB})$, minimum vocal $\mathrm{SPL}(\mathrm{dB})$

Laryngoscopy with stroboscopy using the following standard tasks and measures for laryngeal imaging reporting based on the American Speech and Hearing Association (ASHA) minimum standards for voice assessment.34

The recorded outcomes will be classification of voice disorder (functional neurological (psychogenic), muscle tension voice disorder with laryngeal pathology and muscle tension voice disorder without laryngeal pathology, organic voice disorder).

Tasks Continuous light Strobe light

Rest breathing on three complete breath Vocal fold edge

cycles (inhalation and exhalation)

Laryngeal diadochokinetic task Gross-level vocal fold mobility
/?i?i?i?i?i?i/

Maximum-range vocal fold adduction and Vocal fold mobility maximum range

abduction during alternated/i:/-sniff or

/i:/-quick inhale

Sustained phonation of /i:/ at stable typical pitch and loudness, at least three

Regularity, amplitude, mucosal wave, phase symmetry, vertical level, glottal closure pattern and glottal closure duration 


\section{Table 1 Continued}

Sustained phonation of /i:/ at varied pitches (eg, high and low pitches) of at least three consecutive glottal cycles for each pitch variation

$\begin{array}{ll}\text { Pitch glide } & \text { True vocal fold lengthening } \\ \text { Sustained phonation of /i:/ at varied } & \text { Supraglottic compression }\end{array}$
loudness levels (eg, loud voice and quiet voice production) of at least three consecutive glottal cycles for each loudness variation

\section{Brief conversational speech sample}

Voice therapy task stimulability

$F_{0}$, fundamental frequency; SPL, sound pressure level.

download voice recording software (Audacity, https:// www.audacityteam.org/) or using a mobile phone. The audio file can be sent to the clinician securely using Kiteworks. This recording will form part of the participant's medical record and used for auditory-perceptual and acoustic analysis. Where the participant is unable to record a suitable voice sample at home, a recording will be collected when they attend the clinic for laryngoscopy.

The SLP will then triage the participant for priority of laryngoscopy as described previously and in figure 2 . Laryngoscopy may be provided by an advanced and credentialed SLP in a parallel clinic to the ENT, as previously described in the usual care SLPPC. ${ }^{44}$ After case review of the SLPPC-T data and laryngoscopy imaging, the ENT will provide a diagnosis. The ENT will be blinded to the priority given by the SLP for urgency of laryngoscopy and SLP diagnostic impressions for SLPPC-T.

If participants decline a telehealth assessment, they will be offered usual care through an SLPPC face-to-face consult and will be asked to provide consent for their data to be used in the study for subanalysis. Data collected will include the same protocol as the virtual assessment, with a pseudo-priority rating provided by the SLP before completion of the laryngoscopy. For these participants, the laryngoscopy will be completed on the same day as a one-stop assessment approach.

Figure 3 shows the flow of participants through the study; table 1 shows the assessment protocol; and table 2 outlines the data collection time points. The study infographic in the online supplemental file provides an overview of the SLPPC-T.

Informed consent will be obtained when the participant is contacted by the SLP prior to the telehealth assessment. Evaluation of the outcomes will include only anonymised data collected as part of the initial assessment protocol in line with data collected as part of usual care. Participants will be reminded at their follow-up face-to-face assessment that they are free to withdraw consent at any time, and their data will not be included in the final evaluation.

Table 2 Speech-language pathology primary contact telehealth data collection time points

\begin{tabular}{|c|c|c|c|c|}
\hline \multirow[b]{2}{*}{ Data collection } & \multirow[b]{2}{*}{$\begin{array}{l}\text { Prebaseline (info } \\
\text { from referral) }\end{array}$} & \multicolumn{2}{|l|}{ Baseline } & \multirow[b]{2}{*}{$\begin{array}{l}\text { Face-to-face assessment } \\
\text { (diagnostic laryngoscopy) }\end{array}$} \\
\hline & & Preassessment & $\begin{array}{l}\text { Initial telehealth } \\
\text { assessment }\end{array}$ & \\
\hline Prescreening questionnaire & & $x$ & & \\
\hline Self-report questionnaires & & $x$ & & \\
\hline $\mathrm{PMH}$ and meds & $x$ & $x$ & $x$ & \\
\hline $\begin{array}{l}\text { Primary and secondary symptoms, } \\
\text { variability aggravating factors, } \\
\text { contributing factors }\end{array}$ & $x$ & $x$ & $x$ & \\
\hline Date of symptom onset & $x$ & $x$ & $x$ & \\
\hline Functional voice assessment tasks & & & $x$ & $x$ \\
\hline AQoL-6D ${ }^{+}$ & & $x$ & & \\
\hline VHI-10, RSI, LHQ & & $x$ & & \\
\hline
\end{tabular}

AQOL-6D, Assessment of quality of life-6D; ENT, ear, nose and throat; LHQ, Laryngeal Hypersensitivity Questionnaire; Meds, medications; PMH, previous medical history; RSI, Reflux Symptom Index; SLP, Speech Language Pathologist; VHI-10, Voice Handicap Index. 


\section{Training programme}

Development of an online training programme for SLP and ENT clinicians will include information about the alternative clinical pathway, an assessment manual and data collection tool and fidelity checking process to ensure clinicians are collecting data consistently.

Key areas of the training programme will include

1. Use of the assessment approaches.

2. Decision making around voice disorder classification terminology.

3. Treatment planning decision making.

\section{Data analysis plan}

Descriptive analysis (number, percentages, means and SD) will be used to describe participant demographics, number of participants triaged to each of the laryngoscopy groups, and diagnostic classification before and after laryngoscopy. Logistic regression analysis will be used to examine the association of various signs, symptoms, and voice assessment measures from the SLPPC-T assessment and diagnosis from laryngoscopy assessment. Statistical measures of agreement (weighted kappa statistic) will be used to examine concordance between disorder classification after SLPPC-T assessment and disorder classification after laryngoscopy. Quantitative and qualitative survey data analysis will be used to report the results of the patient experience measures and stakeholder satisfaction surveys. Cost and health outcomes will be measured by obtaining staffing and equipment costs through the GCHHS data analytics team, AQol-6D questionnaire and patient experience surveys, analysed through quantitative and qualitative analysis of survey data. These data will also be used for future economic analysis against a usual care service model. Statistical analysis will be performed using Stata V.16. ${ }^{63}$

\section{Data management}

Quantitative data will be collected at the trial sites using paper clinical records and entered centrally onto the online study database at the trial site and stored on a password-protected computer accessible only by the research team. Qualitative data (audio recordings and video laryngoscopy recordings) will be stored in a secure encrypted online storage database as part of the patients' medical records at the local study site where only the research and clinical team will have password access. Identification logs, screening logs and enrolment logs will be kept at the trial site in a locked cabinet within a secured room.

At the end of the study, all study-related documents will be archived by the coordinating principal investigator for 15 years in line with all relevant legal and statutory requirements.

\section{Study monitoring}

The study will be monitored through the coordinating study group consisting of the coinvestigators, site PI and biostatistician. They will meet regularly during the set-up and duration of the study, data collection and analysis.

The study will monitor and report any adverse events defined as any untoward medical occurrence in a participant as a result of enrolment in the study.

\section{Patient and public involvement}

The concept was designed in response to the need for access to clinical services during a pandemic. Patients were not directly involved in the development of the research. Participants will be offered a results summary at the end of the study.

\section{ETHICS AND DISSEMINATION}

The study has undergone formal ethical review and has been approved by the GCHHS Human Research Ethics Committee (reference number HREC/2020/ QGC/62832). Site-specific approval has been granted by the GCHHS Research Governance Committee (reference number SSA/2020/QGC/62832), where participants will be enrolled for the study. All members of the research team have received training in aspects of good clinical practice in accordance with their role in the study.

Dissemination of the results of the study will be through the publication of articles in peer-reviewed medical journals relevant to health professionals who work with voice disorders and presented at national and international scientific meetings.

\section{STUDY STATUS}

At the time of manuscript submission, the study is open to recruitment.

\section{Author affiliations}

${ }^{1}$ Department of Speech Pathology, Gold Coast Hospital and Health Service, Southport, Queensland, Australia

${ }^{2}$ Faculty of Medicine and Health, The University of Sydney, Sydney, New South Wales, Australia

${ }^{3}$ Department of Ear, Nose and Throat, Gold Coast Hospital and Health Service, Southport, Queensland, Australia

${ }^{4}$ School of Allied Health Sciences, Griffith University Faculty of Health, Gold Coast, Queensland, Australia

${ }^{5}$ Department of Research and Education, Gold Coast Hospital and Health Service, Southport, Queensland, Australia

\section{Twitter Christopher L Payten @chrispaytenSP}

Acknowledgements The authors thank Dr lan Hughes (biostastician at Gold Coast Hospital and Health Service) for his specialist guidance to the data analysis plan for this study and, in addition, Miles Campbell (Dr Liang voice programme education designer and Bridge2Practice Project Manager, The University of Sydney) for the development of the study infographic.

Contributors CLP is the chief investigator and led the concept development and study design, coordinated acquisition of research grant funding and protocol development, and drafted the manuscript. DDN, DN, JO, AMC, KAW and CJM contributed to the study design, grant application and development of the protocol. All authors revised the manuscript and approved the final version.

Funding Data analysis and reporting will be supported by an Allied Health Professions' Office of Queensland Health Practitioner Research Scheme grant (FY 21/22, grant number: not available).

Competing interests None declared. 
Patient consent for publication Not applicable.

Provenance and peer review Not commissioned; externally peer reviewed.

Supplemental material This content has been supplied by the author(s). It has not been vetted by BMJ Publishing Group Limited (BMJ) and may not have been peer-reviewed. Any opinions or recommendations discussed are solely those of the author(s) and are not endorsed by BMJ. BMJ disclaims all liability and responsibility arising from any reliance placed on the content. Where the content includes any translated material, BMJ does not warrant the accuracy and reliability of the translations (including but not limited to local regulations, clinical guidelines, terminology, drug names and drug dosages), and is not responsible for any error and/or omissions arising from translation and adaptation or otherwise.

Open access This is an open access article distributed in accordance with the Creative Commons Attribution Non Commercial (CC BY-NC 4.0) license, which permits others to distribute, remix, adapt, build upon this work non-commercially, and license their derivative works on different terms, provided the original work is properly cited, appropriate credit is given, any changes made indicated, and the use is non-commercial. See: http://creativecommons.org/licenses/by-nc/4.0/.

\section{ORCID iDs}

Christopher L Payten http://orcid.org/0000-0002-4633-6074

Duy Duong Nguyen http://orcid.org/0000-0001-8097-8938

Daniel Novakovic http://orcid.org/0000-0003-3839-1353

Antonia M Chacon http://orcid.org/0000-0003-2902-659X

Kelly A Weir http://orcid.org/0000-0002-5042-1925

Catherine J Madill http://orcid.org/0000-0001-8114-1427

\section{REFERENCES}

1 Kinross P, Suetens C, Gomes Dias J, et al. Rapidly increasing cumulative incidence of coronavirus disease (COVID-19) in the European Union/European economic area and the United Kingdom, 1 January to 15 March 2020. Euro Surveill 2020;25. doi:10.2807/15607917.ES.2020.25.11.2000285. [Epub ahead of print: 16 Mar 2020].

2 World Health organisation (WHO). Coronavirus disease (COVID-19) Dashboard. Available: https://covid19.who.int/ [Accessed Jan 2021].

3 Nicola M, Alsafi Z, Sohrabi C. The socio-economic implications of the coronavirus and COVID-19 pandemic: a review. Int J Surg 2020.

4 Brooks SK, Webster RK, Smith LE, et al. The psychological impact of quarantine and how to reduce it: rapid review of the evidence. Lancet 2020;395:912-20.

5 Ayittey FK, Ayittey MK, Chiwero NB, et al. Economic impacts of Wuhan 2019-nCoV on China and the world. J Med Virol 2020;92:473-5.

6 Lai C-C, Wang C-Y, Wang Y-H, et al. Global epidemiology of coronavirus disease 2019 (COVID-19): disease incidence, daily cumulative index, mortality, and their association with country healthcare resources and economic status. Int $J$ Antimicrob Agents 2020:55:105946.

7 Sahu KK, Mishra AK, Lal A. COVID-2019: update on epidemiology, disease spread and management. Monaldi Arch Chest Dis 2020;90. doi:10.4081/monaldi.2020.1292. [Epub ahead of print: 16 Apr 2020].

8 Arshad Ali S, Baloch M, Ahmed N, et al. The outbreak of Coronavirus Disease 2019 (COVID-19)-An emerging global health threat. J Infect Public Health 2020;13:644-6.

9 Clarke AL, Stephens AF, Liao S, et al. Coping with COVID-19: ventilator splitting with differential driving pressures using standard Hospital equipment. Anaesthesia 2020;75:872-880.

10 Ranney ML, Griffeth V, Jha AK. Critical Supply Shortages - The Need for Ventilators and Personal Protective Equipment during the Covid-19 Pandemic. N Engl J Med 2020;382:e41.

11 World Health Organisation (WHO). SARS-CoV-2 variants. Available: https://www.who.int/csr/don/31-december-2020-sars-cov2-variants/ en/ [Accessed Jan 2021].

12 Ford N, Vitoria M, Rangaraj A, et al. Systematic review of the efficacy and safety of antiretroviral drugs against SARS, MERS or COVID-19: initial assessment. J Int AIDS Soc 2020;23:e25489.

13 Bruinen de Bruin Y, Lequarre A-S, McCourt J, et al. Initial impacts of global risk mitigation measures taken during the combatting of the COVID-19 pandemic. Saf Sci 2020;128:104773.

14 World Health organisation (WHO). Coronavirus disease (COVID-19) advice for the public. Available: https://www.who.int/emergencies/ diseases/novel-coronavirus-2019/advice-for-public [Accessed Jan 2021].

15 Ahn D-G, Shin H-J, Kim M-H, et al. Current status of epidemiology, diagnosis, therapeutics, and vaccines for novel coronavirus disease 2019 (COVID-19). J Microbiol Biotechnol 2020;30:313-24.
16 Jeyanathan M, Afkhami S, Smaill F, et al. Immunological considerations for COVID-19 vaccine strategies. Nat Rev Immunol 2020;20:615-32.

17 Wölfel R, Corman VM, Guggemos W, et al. Virological assessment of hospitalized patients with COVID-2019. Nature 2020;581:465-9.

18 Yu F, Yan L, Wang N, et al. Quantitative detection and viral load analysis of SARS-CoV-2 in infected patients. Clin Infect Dis 2020;71:793-798.

19 American Speech and Hearing Association (ASHA). Guidance to SLPs regarding aerosol generating procedures. Available: https:// www.asha.org/SLP/healthcare/ASHA-Guidance-to-SLPs-RegardingAerosol-Generating-Procedures/ [Accessed Jan 2021].

20 Tran K, Cimon K, Severn M, et al. Aerosol generating procedures and risk of transmission of acute respiratory infections to healthcare workers: a systematic review. PLoS One 2012;7:e35797.

21 The Australian Society for Otolaryngology Head and Neck Surgery. ASOHNS Guidelines Addressing the COVID-19 Pandemic - The Re-introduction of Elective Surgery, 2020. Available: http://asohns. org.au/about-us/news-and-announcements/latest-news?article=88 [Accessed Jan 2021].

22 British Laryngological Association (BLA). Bla and ENT UK Guidleines (endorsed by RCSLT): a graduated return to elective acticities for laryngology within the COVID-19 pandemic, 2020. Available: https://www.britishlaryngological.org/news/bla-ent-uk-guidelinesendorsed-rcslt-graduated-return-elective-activites-laryngology-within [Accessed Jan 2021].

23 American Academy of Otolaryngology-head and Neck Surgery (AAoA-HaN). Guidance for return to practice for otolaryngology-head and neck surgery part one, 2020. Available: https://www.entnet.org/ sites/default/files/guidance_for_return_to_practice_part_1_final_ 050520.pdf [Accessed Jan 2021].

24 Russell A, Oates J, Greenwood K. Prevalence of self-reported voice problems in the general population in South Australia. Am J Speech Lang Pathol 2005;7:24-30.

25 Merrill RM, Roy N, Lowe J. Voice-Related symptoms and their effects on quality of life. Ann Otol Rhinol Laryngol 2013;122:404-11.

26 Benninger MS, Holy CE, Bryson PC, et al. Prevalence and occupation of patients presenting with dysphonia in the United States. J Voice 2017;31:594-600.

27 Jacobson $\mathrm{BH}$, Johnson A, Grywalski C. The voice handicap index (VHI): development and validation. Am J Speech Lang Pathol 1997;6:66-9.

28 Scott S, Robinson K, Wilson JA, et al. Patient-Reported problems associated with dysphonia. Clin Otolaryngol Allied Sci 1997;22:37-40.

29 Cohen SM, Kim J, Roy N, et al. Delayed otolaryngology referral for voice disorders increases health care costs. Am J Med 2015;128:426.e11-8

30 Stachler RJ, Francis DO, Schwartz SR, et al. Clinical practice guideline: hoarseness (dysphonia) (update). Otolaryngol Head Neck Surg 2018;158:S1-42.

31 Koufman JA, Blalock PD. Functional voice disorders. Otolaryngol Clin North Am 1991;24:1059-73.

32 Nouraei SAR, Middleton SE, Butler CR, et al. An estimation of the population incidence of adult unilateral vocal fold mobility impairment in England. Logoped Phoniatr Vocol 2015;40:93-4.

33 Cohen SM, Kim J, Roy N, et al. Prevalence and causes of dysphonia in a large treatment-seeking population. Laryngoscope 2012;122:343-8.

34 Patel RR, Awan SN, Barkmeier-Kraemer J, et al. Recommended protocols for instrumental assessment of voice: American SpeechLanguage-Hearing association expert panel to develop a protocol for instrumental assessment of vocal function. Am J Speech Lang Pathol 2018;27:887-905.

35 Dejonckere $\mathrm{PH}$, Bradley $\mathrm{P}$, Clemente $\mathrm{P}$, et al. A basic protocol for functional assessment of voice pathology, especially for investigating the efficacy of (phonosurgical) treatments and evaluating new assessment techniques. Eur Arch Oto-Rhino-L 2001;258:77-82.

36 Patterson JM, Govender R, Roe J, et al. COVID-19 and ENT SLT services, workforce and research in the UK: a discussion paper. Int $J$ Lang Commun Disord 2020;55:806-17.

37 Paleri V, Hardman J, Tikka T, et al. Rapid implementation of an evidence-based remote triaging system for assessment of suspected referrals and patients with head and neck cancer on follow-up after treatment during the COVID-19 pandemic: model for international collaboration. Head Neck 2020;42:1674-80.

38 Saxon RL, Gray MA, Oprescu FI. Extended roles for allied health professionals: an updated systematic review of the evidence. $J$ Multidiscip Healthc 2014;7:479-88

39 Bornhöft L, Larsson MEH, Thorn J. Physiotherapy in Primary Care Triage - the effects on utilization of medical services at primary 
health care clinics by patients and sub-groups of patients with musculoskeletal disorders: a case-control study. Physiother Theory Pract 2015;31:45-52.

40 Burn D, Beeson E. Orthopaedic triage: cost effectiveness, diagnostic/ surgical and management rates. Clin Gov 2014;19:126-36.

41 Howard Z, Jackman A, Bongers M. Outcomes of a physiotherapy-led pelvic health clinic. Aust N Z J Psychiatry 2018;24:43-50.

42 Carding P. Voice pathology clinics in the UK. Clin Otolaryngol Allied Sci 2003;28:477-8.

43 Seabrook M, Schwarz M, Ward EC, et al. Implementation of an extended scope of practice speech-language pathology allied health practitioner service: an evaluation of service impacts and outcomes. Int J Speech Lang Pathol 2019;21:65-74.

44 Payten CL, Eakin J, Smith T, et al. Outcomes of a multidisciplinary ear, nose and throat allied health primary contact outpatient assessment service. Clin Otolaryngol 2020;45:904-13.

45 Schwarz M, Ward EC, Seabrook M, et al. Outcomes from an extended scope of practice speech-language pathology service for low risk ENT outpatients: a 5-year service review. Int J Speech Lang Pathol 2021:1-9.

46 Mashima PA, Brown JE. Remote management of voice and swallowing disorders. Otolaryngol Clin North Am 2011;44:1305-16.

47 Constantinescu G, Theodoros D, Russell T, et al. Assessing disordered speech and voice in Parkinson's disease: a telerehabilitation application. Int J Lang Commun Disord 2010;45:630-44.

48 Duffy JR, Werven GW, Aronson AE. Telemedicine and the diagnosis of speech and language disorders. Mayo Clin Proc 1997;72:1116-22.

49 Mashima PA, Birkmire-Peters DP, Syms MJ, et al. Telehealth: voice therapy using telecommunications technology. Am J Speech Lang Pathol 2003;12:432-9.

50 Queensalnd Government,. Queensland health clinical prioritisation criteria, 2019. Available: < https://cpc.health.qld.gov.au/Condition/ 67/dysphonia> [Accessed Jan 2021].

51 Roy N, Merrill RM, Gray SD, et al. Voice disorders in the general population: prevalence, risk factors, and occupational impact. Laryngoscope 2005;115:1988-95.
52 Bainbridge KE, Roy N, Losonczy KG, et al. Voice disorders and associated risk markers among young adults in the United States. Laryngoscope 2017;127:2093-9.

53 Bujang MA, Baharum N. Guidelines of the minimum sample size requirements for kappa agreement test. Epidemiol Biostat Public Health 2017;14.

54 Boersma P, Weenink D. Praat: doing phonetics by computer [Computer program], Version 6.1.38, 2021. Available: https://www. fon.hum.uva.nl/praat/ [Accessed Jan 2021].

55 Sprecher A, Olszewski A, Jiang JJ, et al. Updating signal typing in voice: addition of type 4 signals. J Acoust Soc Am 2010;127:3710-6.

56 ENT-UK. 2-Week wait telephone triage. Available: https:// entintegrate.co.uk/entuk2wwtt [Accessed Jan 2021]

57 Kempster GB, Gerratt BR, Verdolini Abbott K, et al. Consensus auditory-perceptual evaluation of voice: development of a standardized clinical protocol. Am J Speech Lang Pathol 2009;18:124-32.

58 Rosen CA, Lee AS, Osborne J, et al. Development and validation of the voice handicap index-10. Laryngoscope 2004;114:1549-56.

59 Belafsky PC, Postma GN, Koufman JA. Validity and reliability of the reflux symptom index (RSI). J Voice 2002;16:274-7.

60 Vertigan AE, Bone SL, Gibson PG. Development and validation of the Newcastle laryngeal hypersensitivity questionnaire. Cough 2014;10:1.

61 Maxwell A, Özmen M, lezzi A, et al. Deriving population norms for the AQoL-6D and AQoL-8D multi-attribute utility instruments from webbased data. Qual Life Res 2016;25:3209-19.

62 Baker J, Ben-Tovim DI, Butcher A, et al. Development of a modified diagnostic classification system for voice disorders with inter-rater reliability study. Logoped Phoniatr Vocol 2007;32:99-112.

63 StataCorp. Stata statistical software: release 16. College Station, TX: StataCorp LLC, 2019.

64 Fairbanks G. Voice and articulation drillbook. 2nd edn. New York: Harper \& Row, 1960: 124-39. 